\title{
CHEBYSHEV-TYPE QUADRATURE AND PARTIAL SUMS OF THE EXPONENTIAL SERIES
}

\author{
ARNO KUIJLAARS
}

ABSTRACT. Chebyshev-type quadrature for the weight functions

$$
w_{a}(t)=\frac{1-a t}{\pi \sqrt{1-t^{2}}}, \quad-1<t<1, \quad-1<a<1,
$$

is related to a problem concerning partial sums of the exponential series, namely the problem to extend the $n$th partial sum to a polynomial of degree $2 N$ having all zeros on the circle $|z|=|a| N$. Using this connection, we show that the minimal number $N$ of nodes needed for Chebyshev-type quadrature of degree $n$ for $w_{a}(t)$ satisfies an inequality $C_{1} n \leq N \leq C_{2} n$ with positive constants $C_{1}, C_{2}$. As an application we prove that the minimal number $N$ of nodes for Chebyshev-type quadrature of degree $n$ on a torus embedded in $\mathbf{R}^{3}$ satisfies an inequality $C_{1} n^{2} \leq N \leq C_{2} n^{2}$.

\section{INTRODUCTION AND STATEMENT OF RESULTS}

A Chebyshev-type quadrature formula is a numerical integration formula in which all weights are equal. For an integrable nonnegative weight function $w(t)$ on $[-1,1]$ with $\int_{-1}^{1} w(t) d t=1$, this is a formula of the type

$$
\int_{-1}^{1} f(t) w(t) d t \approx \frac{1}{N} \sum_{i=1}^{N} f\left(x_{i}\right)
$$

with (not necessarily distinct) nodes $x_{i} \in[-1,1], i=1, \ldots, N$. We call $N$ the size of (1.1). The degree of (1.1) is the maximal number $n$ such that equality holds for every polynomial $f(t)$ of degree $\leq n$. We say that $w(t)$ admits Chebyshev-type quadrature of size $N$ and degree $n$ if there exist $N$ points $x_{i} \in[-1,1]$ such that $(1.1)$ has degree $n$. See [2, 3], for surveys on Chebyshev-type quadrature.

If $N \leq n$, then (1.1) is called a Chebyshev quadrature formula. We say that $w(t)$ admits Chebyshev quadrature if a Chebyshev quadrature formula exists for every $n$. The classical example of a weight function which admits

Received by the editor April 22, 1993 and, in revised form, October 27, 1993.

1991 Mathematics Subject Classification. Primary 65D32; Secondary 30C15, 41A55.

Key words and phrases. Chebyshev-type quadrature, partial sums, distribution of zeros, multidimensional quadrature.

Supported (in part) by the Netherlands Foundation for Mathematics SMC with financial aid from the Netherlands Organization for the Advancement of Scientific Research (NWO). 
Chebyshev quadrature is the function $\left(1-t^{2}\right)^{-1 / 2} / \pi$, but more examples are known; see [2] and the references therein.

In this paper we consider the weight functions

$$
w_{a}(t)=\frac{1-a t}{\pi \sqrt{1-t^{2}}}, \quad-1<t<1, \quad-1 \leq a \leq 1
$$

These functions arise in connection with quadrature problems on the surface of a torus; see $\S 5$. It has been proved by Xu [16] that $w_{a}(t)$ admits Chebyshev quadrature if $|a|<\gamma=0.27846 \ldots$, where $\gamma$ is the unique positive root of $x e^{1+x}=1$.

We show that for the weight functions $w_{a}(t)$, the existence of Chebyshevtype quadrature is related to properties of the partial sums $s_{n}(z)$ of the exponential series,

$$
s_{n}(z)=\sum_{k=0}^{n} \frac{z^{k}}{k !} .
$$

Using a general condition for the existence of Chebyshev-type quadrature (Theorem 1), we find that $w_{a}(t)$ admits Chebyshev-type quadrature of size $N$ and degree $\geq n$ if and only if $s_{n}(z)$ can be extended to a real polynomial of degree $2 N$ having all its zeros on the circle $|z|=|a| N$. Here we say that $p(z)$ is an extension of $s_{n}(z)$ if $p(z)=s_{n}(z)+\mathscr{O}\left(z^{n+1}\right) \quad(z \rightarrow 0)$. Furthermore, if $s_{n}(z)$ has an extension to a polynomial of degree $2 N-n-1$ which has all its zeros in $|z|>|a| N$, then $w_{a}(t)$ admits Chebyshev-type quadrature of size $N$ and degree $\geq n$.

Thus, we are led to consider extensions of $s_{n}(z)$ which have their zeros as far from the origin as possible. Our main results are as follows:

- For $0<R<\frac{1}{2}$ there is a constant $c$ such that every $s_{n}(z)$ has an extension to a polynomial of degree $c n$ which has no zeros in $|z|<R c n$ (Theorem 6).

- For $a \in(-1,1)$ there exist positive constants $C_{1}, C_{2}$ such that $w_{a}(t)$ admits Chebyshev-type quadrature of degree $n$ and size $N$ where

$$
C_{1} n \leq N \leq C_{2} n
$$

(Corollary 7). An application to Chebyshev-type quadrature on the surface of the torus is given in Theorem 8.

- For $|a|=1$ the corresponding bounds are

$$
C_{1} n^{3} \leq N \leq C_{2} n^{3}
$$

which imply that $s_{n}(z)$ can be extended to a polynomial of degree $N \approx$ $\mathrm{Cn}^{3}$ which has all its zeros on the circle $|z|=N / 2$ (Corollary 5).

- The bound $|a|<\gamma$ for the existence of Chebyshev quadrature is sharp: For $|a|>\gamma$, the weight function $w_{a}(t)$ does not admit Chebyshev quadrature (Proposition 4). 


\section{CONDITION FOR CHEBySheV-TYPE QUADRATURE}

Let $w(t)$ be a weight function on $[-1,1]$ with $\int_{-1}^{1} w(t) d t=1$. Set

$$
c_{k}=2 \int_{-1}^{1} T_{k}(t) w(t) d t, \quad k \geq 1,
$$

where $T_{k}(t)$ is the Chebyshev polynomial of the first kind of degree $k$, and construct the power series

$$
G(z)=\sum_{k=1}^{\infty} \frac{c_{k}}{k} z^{k}
$$

which is analytic in $|z|<1$. In view of the formula

$$
-\log \left(1-2 t z+z^{2}\right)=\sum_{k=1}^{\infty} \frac{2}{k} T_{k}(t) z^{k},
$$

cf. [14, equation (4.7.25)], we see that

$$
G(z)=-\int_{-1}^{1} \log \left(1-2 t z+z^{2}\right) w(t) d t .
$$

In terms of the function $G(z)$ we have the following conditions for the existence of Chebyshev-type quadrature. Theorem 1 is a slight modification of results due to Geronimus [4, Theorem 1] and Peherstorfer [8, Theorem 1], [9, Theorem 3]. For convenience of the reader we have included the proof.

Theorem 1. Let $w(t)$ be a nonnegative integrable function on $[-1,1]$ such that $\int_{-1}^{1} w(t) d t=1$. Let $G(z)$ be defined by (2.1) and (2.2). Let $n, N \in \mathbf{N}$. Then the following hold:

1. The weight function $w(t)$ admits Chebyshev-type quadrature of size $N$ and degree $\geq n$ with all nodes in the open interval $(-1,1)$ if and only if there is a real polynomial $P(z)$ of degree $2 N$ such that

(a) $P(z)=\exp (-N G(z))+\mathscr{O}\left(z^{n+1}\right) \quad(z \rightarrow 0)$,

(b) all zeros of $P(z)$ are nonreal and have modulus 1 .

2. If there is a real polynomial $p(z)$ of degree $2 N-n-1$ such that

(a) $p(z)=\exp (-N G(z))+\mathscr{O}\left(z^{n+1}\right) \quad(z \rightarrow 0)$,

(b) all zeros of $p(z)$ have modulus $>1$,

then $w(t)$ admits Chebyshev-type quadrature of size $N$ and degree $\geq n$.

Proof. Suppose $P(z)$ satisfies 1.(a)(b), so that $P(0)=1$ and all zeros of $P(z)$ are complex and come in conjugate pairs. Then there are $\phi_{j} \in(0, \pi), j=$ $1, \ldots, N$, such that

$$
P(z)=\prod_{j=1}^{N}\left(e^{i \phi_{j}}-z\right)\left(e^{-i \phi_{j}}-z\right) .
$$


We will compute the logarithmic derivative of $P(z)$ in two ways. From (a) and (2.2) we have

$$
\frac{P^{\prime}(z)}{P(z)}=-N G^{\prime}(z)+\mathscr{O}\left(z^{n}\right)=-N \sum_{k=1}^{n} c_{k} z^{k-1}+\mathscr{O}\left(z^{n}\right) \quad(z \rightarrow 0) .
$$

From (2.3) it follows that

$$
\begin{aligned}
\frac{P^{\prime}(z)}{P(z)} & =-\sum_{j=1}^{N}\left[\frac{1}{e^{i \phi_{j}}-z}+\frac{1}{e^{-i \phi_{j}}-z}\right]=-2 \sum_{j=1}^{N} \sum_{k=1}^{\infty} \cos \left(k \phi_{j}\right) z^{k-1} \\
& =-2 \sum_{k=1}^{\infty} \sum_{j=1}^{N} T_{k}\left(x_{j}\right) z^{k-1}
\end{aligned}
$$

where we have written $\cos \phi_{j}=x_{j}$.

Comparing coefficients in (2.4) and (2.5) and using (2.1), we find

$$
\frac{1}{N} \sum_{j=1}^{N} T_{k}\left(x_{j}\right)=\frac{c_{k}}{2}=\int_{-1}^{1} T_{k}(t) w(t) d t, \quad k=1, \ldots, n,
$$

that is, the points $x_{j} \in(-1,1), j=1, \ldots, N$, are the nodes of a Chebyshevtype quadrature formula for $w(t)$ of degree $\geq n$.

Conversely, if $x_{j} \in(-1,1), j=1, \ldots, N$, are the nodes of a Chebyshevtype quadrature formula of degree $\geq n$, then writing $x_{j}=\cos \phi_{j}$ and defining $P(z)$ as in equation (2.3), we can easily check that $P(z)$ satisfies $1 .(\mathrm{a})(\mathrm{b})$.

Next, assume that the real polynomial $p(z)$ of degree $2 N-n-1$ satisfies 2.(a)(b). Let $p^{*}(z)=z^{2 N-n-1} p\left(z^{-1}\right)$ denote the reciprocal polynomial of $p(z)$. Then

$$
P(z):=p(z)+z^{n+1} p^{*}(z)
$$

is a real polynomial of degree $2 N$ (exactly) which has all its zeros on the unit circle, cf. [10, pp. 88 and 256] for a related result of Schur. Note that $P( \pm 1)=$ $2 p( \pm 1) \neq 0$, so that $P(z)$ satisfies condition 1.(b). Since $p(z)$ satisfies 2 .(a), it is clear from the definition of $P(z)$ that $P(z)$ satisfies 1 .(a) and the theorem follows.

\section{The Weight FUnCtions $w_{a}(t)$}

For the weight function

$$
w_{a}(t)=\frac{1-a t}{\pi \sqrt{1-t^{2}}}, \quad t \in(-1,1), \quad-1 \leq a \leq 1,
$$

the function $G(z)$ of formula (2.2) is simply $G(z)=-a z$, and the condition 2.(a) of Theorem 1 is

$$
p(z)=\exp (a N z)+\mathscr{O}\left(z^{n+1}\right) \quad(z \rightarrow 0) .
$$

Denoting by $s_{n}(z)=\sum_{j=0}^{n} z^{j} / j$ ! the $n$th partial sum of the exponential series, we obtain for $a \neq 0$,

$$
p(z / a N)=s_{n}(z)+\mathscr{O}\left(z^{n+1}\right) \quad(z \rightarrow 0) .
$$


A result of Seymour and Zaslavsky [11, Corollary 2] shows that for every $n$, Chebyshev-type quadrature formulas of degree $\geq n$ exist in case the size $N$ is sufficiently large. So part 1 of Theorem 1 implies

Corollary 2. Let $n \in \mathbf{N}, 0<a \leq 1$. For $N$ sufficiently large, $s_{n}(z)$ has an extension to a real polynomial of degree $2 N$ having all its zeros on the circle $|z|=a N$.

Note that the bound $a \leq 1$ is sharp. For $a>1$, it is not possible that every $s_{n}(z)$ has an extension to a real polynomial of degree $2 N$ having all its zeros on $|z|=a N$, since that would imply that Chebyshev-type quadrature of every degree exists for the weight function $(1-a t) /\left(\pi \sqrt{1-t^{2}}\right)$ which assumes negative values in $(-1,1)$. This is impossible, since a slim high-peaked impulse function, centered at a point where the weight function is negative could be approximated arbitrarily closely by a polynomial of sufficiently high degree whose square could then be taken in the role of $f$ in (1.1). This would produce a negative number on the left, and a nonnegative number on the right.

Part 2 of Theorem 1 gives the following condition for the existence of a Chebyshev-type quadrature for $w_{a}(t)$.

Corollary 3. Let $-1 \leq a \leq 1$. If $s_{n}(z)$ has an extension to a polynomial of degree $2 N-n-1$ which has all its zeros in $|z|>|a| N$, then there exists a Chebyshev-type quadrature formula for $w_{a}(t)$ of size $N$ and degree $\geq n$.

The question of Chebyshev quadrature for $w_{a}(t)$ has been discussed by $\mathrm{Xu}$ [16]. He proved that $w_{a}(t)$ admits Chebyshev quadrature if $|a|<\gamma=$ $0.2784645 \ldots$, where $\gamma$ is the unique positive solution of $x e^{1+x}=1$.

Corollary 3 with $N=n+1$ shows that Chebyshev-type quadrature of size $n+1$ and degree $\geq n$ is possible if the zeros of $s_{n+1}(z)$ have absolute value $>|a|(n+1)$. [Take $s_{n+1}(z)$ as the extension of $s_{n}(z)$.] The behavior of the zeros of $s_{n}(z)$ has been well studied. It is a classical result of Szegö [13] that accumulation points of the zeros of the normalized partial sums $s_{n}(n z)$ lie on the curve given by

$$
\left|e^{1-z} z\right|=1, \quad|z| \leq 1 .
$$

Later, Buckholtz [1] showed that all zeros lie outside this curve. The point on the curve with smallest absolute value is on the negative real axis and is $-\gamma$, which is in accordance with Xu's result. For more details on the zeros of $s_{n}(z)$, see [15, Chapter 4].

Using Theorem 1, we can prove that Xu's bound $|a|<\gamma$ for the existence of Chebyshev quadrature is sharp.

Proposition 4. For $|a|>\gamma$, the weight function $w_{a}(t)$ does not admit Chebyshev quadrature.

Proof. Without loss of generality we take $a \in(\gamma, 1)$.

Let $n \in \mathbf{N}$ and suppose that $w_{a}(t)$ admits Chebyshev quadrature of degree $n$. By part 1 of Theorem 1 there is a real polynomial $P(z)$ of degree $2 n$ having all its zeros on the unit circle and satisfying

$$
P(z)=\exp (a n z)+\mathscr{O}\left(z^{n+1}\right) \quad(z \rightarrow 0) .
$$


Then $P(z)=P^{*}(z)$ and it easily follows that

$$
P(z)=q_{n}(z)+z^{n} q_{n}^{*}(z)
$$

with

$$
q_{n}(z)=\sum_{k=0}^{n-1} \frac{(a n z)^{k}}{k !}+\frac{1}{2} \frac{(a n z)^{n}}{n !}=\frac{1}{2}\left(s_{n}(\text { anz } z)+s_{n-1}(\text { an } z)\right) .
$$

In particular, $P(1)=2 q_{n}(1)>0$ and $P(-1)=2 q_{n}(-1)$. If we could show that $q_{n}(-1)<0$, then it would follow that $P(z)$ has a zero in the interval $(-1,1)$, which would be a contradiction. Therefore we will show that $q_{n}(-1)<0$ for $n$ sufficiently large (in fact only for $n$ even).

Since for $z \in \mathbf{C}$,

$$
e^{-z} s_{n}(z)=1-\frac{1}{n !} \int_{0}^{z} e^{-t} t^{n} d t
$$

(which can be verified by differentiation), it follows that

$$
\begin{aligned}
\frac{1}{2} e^{-z}\left(s_{n}(z)+s_{n-1}(z)\right) & =1-\frac{1}{2 n !} \int_{0}^{z} e^{-t}\left(t^{n}+n t^{n-1}\right) d t \\
& =1-\frac{(-1)^{n} n^{n+1}}{2 n !} \int_{0}^{-z / n} e^{n x}\left(x^{n-1}-x^{n}\right) d x,
\end{aligned}
$$

where we have made the substitution $t=-n x$. Hence

$$
e^{-a n z} q_{n}(z)=1-\frac{(-1)^{n} n^{n+1}}{2 n !} e^{-n} \int_{0}^{-a z} e^{(1+x) n} x^{n}\left(\frac{1}{x}-1\right) d x
$$

and we see that $q_{n}(-1)<0$ if and only if $n$ is even and

$$
\int_{0}^{a} e^{(1+x) n} x^{n}\left(\frac{1}{x}-1\right) d x>\frac{2 n ! e^{n}}{n^{n+1}} .
$$

For the left-hand side we have

$$
\int_{0}^{a} e^{(1+x) n} x^{n}\left(\frac{1}{x}-1\right) d x>\left(\frac{1}{a}-1\right) \int_{0}^{a}\left(e^{1+x} x\right)^{n} d x .
$$

Since $a>\gamma$ we have $e^{1+a} a>1$ and we see that that the left-hand side of (3.1) increases exponentially as $n \rightarrow \infty$. Further, the right-hand side tends to 0 for $n \rightarrow \infty$, so that for $n$ large enough the inequality (3.1) holds and the proposition follows.

For the special cases $a= \pm 1$, the weight function $w_{a}(t)$ is a Jacobi weight function. Chebyshev-type quadrature for $w_{a}(t)$ is related to Chebyshev-type quadrature for the ultraspherical weight function $2\left(1-s^{2}\right)^{1 / 2} / \pi$ because of the relation

$$
\frac{1}{\pi} \int_{-1}^{1} f(t)(1-t)^{1 / 2}(1+t)^{-1 / 2} d t=\frac{2}{\pi} \int_{-1}^{1} f\left(2 s^{2}-1\right)\left(1-s^{2}\right)^{1 / 2} d s .
$$

[We have taken $a=+1$.] Using this relation and the symmetry of the weight function $2\left(1-s^{2}\right)^{1 / 2} / \pi$ we get the following: 
If $x_{1}, \ldots, x_{N}$ are the nodes of a Chebyshev-type quadrature formula for $w_{1}(t)$ of degree $n$ then the $2 N$ points

$$
\pm\left(\frac{x_{1}+1}{2}\right)^{1 / 2}, \pm\left(\frac{x_{2}+1}{2}\right)^{1 / 2}, \ldots, \pm\left(\frac{x_{N}+1}{2}\right)^{1 / 2}
$$

are the nodes of a Chebyshev-type quadrature formula for $2\left(1-s^{2}\right)^{1 / 2} / \pi$ of degree $2 n+1$.

Conversely, if $\pm y_{1}, \ldots, \pm y_{N}$ are the nodes of a symmetric Chebyshev-type quadrature formula for $2\left(1-s^{2}\right)^{1 / 2} / \pi$ of degree $2 n+1$, then

$$
2 y_{1}^{2}-1,2 y_{2}^{2}-1, \ldots, 2 y_{N}^{2}-1
$$

are the nodes of a Chebyshev-type quadrature formula of degree $n$ for $w_{1}(t)$.

For the weight function $2\left(1-s^{2}\right)^{1 / 2} / \pi$, the author [7] has shown that the minimal number $N$ of nodes needed for Chebyshev-type quadrature of degree $n$ satisfies an inequality

$$
C_{1} n^{3} \leq N \leq C_{2} n^{3},
$$

where $C_{1}, C_{2}$ are positive constants which do not depend on $n$. Hence also for $w_{1}(t)$, Chebyshev-type quadrature of degree $n$ is possible with $\approx C n^{3}$ nodes, and this is the correct order. Now part 1 of Theorem 1 immediately gives:

Corollary 5. There exist constants $C_{1}, C_{2}>0$ such that, for every $n \in \mathbf{N}, s_{n}(z)$ has an extension to a polynomial of degree $N$ with $C_{1} n^{3} \leq N \leq C_{2} n^{3}$ whose zeros are nonreal and all lie on the circle $|z|=N / 2$. The order $n^{3}$ cannot be improved.

For $\gamma<|a|<1$ no results on Chebyshev-type quadrature seem to be known. We will show that the minimal number of nodes $N$ needed for Chebyshev-type quadrature of degree $n$ for $w_{a}(t)$ satisfies an inequality $C_{1} n \leq N \leq C_{2} n$. The positive constants $C_{1}$ and $C_{2}$ depend on $a$ but not on $n$. To obtain this result, we will construct extensions of $s_{n}(z)$.

\section{EXTENSION OF PARTIAL SUMS OF THE EXPONENTIAL SERIES}

We will prove the following theorem.

Theorem 6. Let $0<R<\frac{1}{2}$. Then there is a constant $c_{0}=c_{0}(R) \in \mathbf{N}$ such that, for every $n$ and every $c \geq c_{0}, s_{n}(z)$ has an extension to a polynomial of degree cn which is zero-free in the disc $|z|<R c n$.

Remark. a) From the results of Szegö [13] and Buckholtz [1] (see §3) it follows that one can take $c_{0}=1$ in case $R<\gamma=0.2784645 \ldots$.

b) The theorem does not hold for $R \geq \frac{1}{2}$; see Corollary 5 .

Proof. Motivated by the relation

$$
e^{-z} s_{n}(z)=1-\frac{1}{n !} \int_{0}^{z} e^{-t} t^{n} d t,
$$

we will study polynomials $S_{N}(z)$ satisfying

$$
e^{-z} S_{N}(z)=1-\frac{1}{A_{n}} \int_{0}^{z} e^{-t} t^{n} p_{m}(t)^{n} d t .
$$


Here $p_{m}(t)$ will be a polynomial of degree $m$ and

$$
A_{n}=\int_{0}^{\infty} e^{-t} t^{n} p_{m}(t)^{n} d t .
$$

It is easy to see that with this choice of $A_{n},(4.1)$ defines a polynomial $S_{N}(z)$ of degree $N:=(1+m) n$ and

$$
S_{N}(z)=s_{n}(z)+\mathscr{O}\left(z^{n+1}\right) \quad(z \rightarrow 0) .
$$

Thus, $S_{N}(z)$ is an extension of $s_{n}(z)$ to a polynomial of degree $(1+m) n$.

For $p_{m}(t)$ we take $q_{m}(t / N)$, where $q_{m}(w)$ is a monic polynomial with real coefficients. In the integrals of (4.1) and (4.2) we make the substitution $t=w N$ to obtain

$$
e^{-z} S_{N}(z)=1-\frac{1}{B_{n}} \int_{0}^{z / N}\left[e^{-(1+m) w} w q_{m}(w)\right]^{n} d w
$$

with

$$
B_{n}=\int_{0}^{\infty}\left[e^{-(1+m) w} w q_{m}(w)\right]^{n} d w .
$$

From (4.3) it is clear that $S_{N}(z)$ is zero-free in the region defined by

$$
\left|\int_{0}^{z / N}\left[e^{-(1+m) w} w q_{m}(w)\right]^{n} d w\right|<B_{n} .
$$

The rest of the proof will be divided into three steps. In Step 1 we introduce an auxiliary function $F(z)$ and establish some basic properties. In Step 2 we define for every $m$ a polynomial $q_{m}(t)$ and a function $F_{m}(z)$. We show that $F_{m}(z)$ tends to $F(z)$ as $m \rightarrow \infty$. Using these results we will show in Step 3 that for $m$ large enough (say $m \geq m_{0}$ ) the inequality (4.5) holds for every $n \in \mathbf{N}$ and for every $|z|<R N=R(1+m) n$.

Then the theorem follows with $c_{0}=1+m_{0}$.

Step 1. Take $r=2 R^{2}$ so that $0<r<R<1 / 2$ and let $\rho$ be the measure on the circle $\xi=r e^{i \theta}$ given by

$$
d \rho(\xi)=\frac{1-\cos \theta}{2 \pi} d \theta .
$$

The moments of $\rho$ are easily computed:

$$
\int \xi^{k} d \rho(\xi)=\left\{\begin{array}{cc}
1 & \text { for } k=0 \\
-r / 2 & \text { for } k=1 \\
0 & \text { for } k \geq 2
\end{array}\right.
$$

Define for $|z|>r$,

$$
F(z)=-\operatorname{Re} z+\int \log |z-\xi| d \rho(\xi) .
$$


Since for $|z|>r$,

$$
\begin{aligned}
\int \log |z-\xi| d \rho(\xi) & =\log |z|+\operatorname{Re} \int \log \left(1-\frac{\xi}{z}\right) d \rho(\xi) \\
& =\log |z|-\operatorname{Re} \sum_{k=1}^{\infty} \frac{1}{k z^{k}} \int \xi^{k} d \rho(\xi) \\
& =\log |z|+\frac{r}{2} \operatorname{Re} \frac{1}{z}
\end{aligned}
$$

we have

$$
F(z)=-\operatorname{Re} z+\log |z|+\frac{r}{2} \operatorname{Re} \frac{1}{z} .
$$

We need two properties of $F(z)$.

A: $F(z)$ is constant on the circle $|z|=R$.

Indeed, since $\operatorname{Re}(1 / z)=\operatorname{Re} z /|z|^{2}$, we have for $|z|=R$,

$$
F(z)=\log R+\operatorname{Re} z\left[-1+\frac{r}{2 R^{2}}\right]=\log R .
$$

B: $F(x)$ is strictly increasing on the interval $(r, 1 / 2+\epsilon)$, where

$$
\epsilon:=\left(1 / 4-R^{2}\right)^{1 / 2}>0 .
$$

Indeed, using (4.7), we compute for $z=x>r$,

$$
F^{\prime}(x)=-1+\frac{1}{x}-\frac{r}{2 x^{2}}=\frac{-(x-1 / 2)^{2}+\epsilon^{2}}{x^{2}}
$$

and property $B$ follows.

From properties A and B we obtain (recall $r<R<1 / 2$ )

$$
\max _{|z|=R} F(z)<F(1 / 2)
$$

and for some $\delta>0$,

$$
F(1 / 2)+\delta<F(x) \quad \text { for all } x \in(1 / 2+\epsilon / 2,1 / 2+\epsilon) \text {. }
$$

In the rest of the proof, $\epsilon$ as defined in (4.8) and $\delta$ satisfying (4.10) will be fixed.

Step 2. For every $m$, take $m$ points $\xi_{1, m}, \ldots, \xi_{m, m}$ on the circle $|\xi|=r$ as follows. We let $\xi_{j, m}=r e^{i \theta_{j, m}}$, where

$\int_{0}^{\theta_{1, m}} \frac{1-\cos \theta}{2 \pi} d \theta=\frac{1}{2 m}, \quad \int_{\theta j, m}^{\theta_{j+1, m}} \frac{1-\cos \theta}{2 \pi} d \theta=\frac{1}{m}, \quad j=1, \ldots, m-1$.

In this way, we have $\xi_{m+1-j}=\bar{\xi}_{j}$ and no $\xi_{j, m}$ is real and positive. We also define $\xi_{0, m}=0$. Put

$$
q_{m}(z)=\prod_{j=1}^{m}\left(z-\xi_{j, m}\right)
$$


Then $q_{m}(z)$ is a monic polynomial of degree $m$ with real coefficients and $q_{m}(z)>0$ for $z$ real and positive. Let $\rho_{m}$ be the normalized counting measure of the points $\xi_{0, m}, \xi_{1, m}, \ldots, \xi_{m, m}$ :

$$
\rho_{m}=\frac{1}{m+1} \sum_{j=0}^{m} \delta_{\xi_{j, m}} .
$$

The measures $\rho_{m}$ converge to $\rho$ in the weak *-topology for convergence of measures. Write

$$
\begin{aligned}
F_{m}(z) & =-\operatorname{Re} z+\frac{1}{m+1} \log \left|z q_{m}(z)\right| \\
& =-\operatorname{Re} z+\frac{1}{m+1} \sum_{j=0}^{m} \log \left|z-\xi_{j, m}\right| \\
& =-\operatorname{Re} z+\int \log |z-\xi| d \rho_{m}(\xi) .
\end{aligned}
$$

The function $F_{m}(z)$ is subharmonic on $\mathbf{C}$ and is harmonic for $z \neq \xi_{j, m}$, $j=0, \ldots, m$, so in particular, $F_{m}(z)$ is harmonic for $|z|>r$.

Comparing (4.6) and (4.11), we have that

$$
\lim _{m \rightarrow \infty} F_{m}(z)=F(z)
$$

pointwise for $|z|>r$. As the points $\xi_{j, m}, j=0, \ldots, m$, have absolute values $\leq r$, it easily follows from (4.11) that the functions $F_{m}(z)$ are uniformly bounded on compact subsets of $|z|>r$. Since the functions $F_{m}(z)$ are harmonic for $|z|>r$, this implies that they form a normal family (see, e.g., [5, Theorem 2.18]). It follows that the limit (4.12) is uniform on every compact subset of $|z|>r$.

Then by (4.9) and (4.10) we have for all $m$ sufficiently large,

$$
\max _{|z|=R} F_{m}(z)<F(1 / 2),
$$

and

$$
F(1 / 2)+\delta<F_{m}(x) \quad \text { for all } x \in(1 / 2+\epsilon / 2,1 / 2+\epsilon) .
$$

Since $F_{m}(z)$ is subharmonic on $\mathbf{C},(4.13)$ also gives

$$
\max _{|z| \leq R} F_{m}(z)<F(1 / 2) \text {. }
$$

Step 3. We take $m$ such that (4.14), (4.15) hold and such that

$$
e^{(1+m) \delta} \epsilon / 2 \geq R \text {. }
$$

For a given $n \in \mathbf{N}$ we write $N=(1+m) n$ and we are going to prove (4.5) for $|z|<R N$. 
Note that by the definition (4.11)

$$
\left|e^{-(1+m) w} w q_{m}(w)\right|=e^{(1+m) F_{m}(w)} .
$$

Thus, if $|z|<R N$, then by (4.15) and (4.17),

$$
\left|\int_{0}^{z / N}\left[e^{-(1+m) w} w q_{m}(w)\right]^{n} d w\right| \leq \operatorname{Re} N F(1 / 2) .
$$

Also by (4.4), (4.14), (4.17) and the fact that $q_{m}(w)>0$ for $w>0$, we have

$$
B_{n} \geq \int_{1 / 2+\epsilon / 2}^{1 / 2+\epsilon} e^{N F_{m}(w)} d w \geq e^{N(F(1 / 2)+\delta)} \epsilon / 2 .
$$

From (4.18) and (4.19) we see that (4.5) holds for every $|z|<R N$ if $e^{N \delta} \epsilon / 2 \geq$ $R$. Since $N=(1+m) n$, this follows from (4.16).

Corollary 7. For every $a \in(-1,1)$ there exist constants $C_{1}, C_{2}$ depending on $a$, such that, for every $n$, the minimal number $N$ of nodes in a Chebyshev-type quadrature formula of degree $n$ for $w_{a}(t)$ satisfies the inequalities

$$
C_{1} n \leq N \leq C_{2} n \text {. }
$$

Proof. The upper bound follows easily from Theorem 6 and Corollary 3 .

For the lower bound we take $C_{1}=1 / 2$. It is a general result that for any quadrature formula of degree $\geq n$ with arbitrary weights one needs more than $n / 2$ nodes.

\section{CHEBYSHEV-TYPE QUADRATURE ON THE TORUS}

Fix $0<a<1$ and let $T_{a}$ be the torus embedded in $\mathbf{R}^{3}$ with parametrization

$$
\begin{aligned}
& x=\cos \phi(1+a \cos \psi), \\
& y=\sin \phi(1+a \cos \psi), \quad 0 \leq \phi<2 \pi, \quad 0 \leq \psi<2 \pi \\
& z=a \sin \psi,
\end{aligned}
$$

The surface element is $a(1+a \cos \psi) d \phi d \psi=d \sigma$ and the surface area is $4 \pi^{2} a$. A Chebyshev-type quadrature formula for $T_{a}$ is a formula of the form

$$
\frac{1}{4 \pi^{2} a} \iint_{T_{a}} f(x, y, z) d \sigma \approx \frac{1}{N} \sum_{i=1}^{N} f\left(x_{i}, y_{i}, z_{i}\right)
$$

with $\left(x_{i}, y_{i}, z_{i}\right) \in T_{a}$. The degree of $(5.1)$ is the maximal $n$ such that equality holds for all polynomials in three variables $f(x, y, z)$ of total degree $\leq n$.

Multidimensional Chebyshev-type quadrature formulas for various other regions were given by Korevaar and Meyers [6].

Theorem 8. Let $0<a<1$. There exist constants $C_{1}, C_{2}>0$ (depending on $a$ ) such that the minimal number $N$ of nodes needed for Chebyshev-type quadrature on $T_{a}$ of degree $\geq n$ satisfies the inequalities

$$
C_{1} n^{2} \leq N \leq C_{2} n^{2}
$$


Proof. The lower bound follows from a result on general quadrature formulas (i.e., not necessarily with equal weights) for 2-dimensional domains. Let

$$
\frac{1}{4 \pi^{2} a} \iint_{T_{a}} f(x, y, z) d \sigma \approx \sum_{i=1}^{N} \lambda_{i} f\left(x_{i}, y_{i}, z_{i}\right)
$$

be a quadrature formula of degree $n$ with weights $\lambda_{i}$. Then for polynomials $g(x, y)$ of two variables of degree $\leq n$, we have

$$
\iint_{A_{a}} g(x, y) w(x, y) d x d y=\sum_{i=1}^{N} \lambda_{i} g\left(x_{i}, y_{i}\right),
$$

where $A_{a}$ is the annulus

$$
A_{a}:=\left\{(x, y) \mid 1-a \leq \sqrt{x^{2}+y^{2}} \leq 1+a\right\},
$$

and $w(x, y)$ is a positive weight function on $A_{a}$. A result of Stroud [12, Theorem 3.15-1] shows that the number of nodes satisfies $N \geq n^{2} / 8$.

For the upper bound, we first consider polynomials $f(x, y, z)$ of degree $\leq n$ which are even in $y$ and $z$. For such polynomials we have

$$
\begin{aligned}
\frac{1}{4 \pi^{2} a} \iint_{T_{a}} f(x, y, z) d \sigma & =\frac{1}{4 \pi^{2}} \int_{0}^{2 \pi} \int_{0}^{2 \pi} F(\phi, \psi)(1+a \cos \psi) d \phi d \psi \\
& =\frac{1}{\pi^{2}} \int_{0}^{\pi} \int_{0}^{\pi} F(\phi, \psi)(1+a \cos \psi) d \phi d \psi,
\end{aligned}
$$

where we have written

$$
F(\phi, \psi)=f(\cos \phi(1+a \cos \psi), \sin \phi(1+a \cos \psi), a \sin \psi) .
$$

There is a polynomial $p(s, t)$ of degree $\leq 2 n$ such that

$$
p(\cos \phi, \cos \psi)=F(\phi, \psi),
$$

and the substitutions $\cos \phi=s, \cos \psi=t$ give

$$
\frac{1}{4 \pi^{2} a} \iint_{T_{a}} f(x, y, z) d \sigma=\int_{-1}^{1} \int_{-1}^{1} p(s, t) \frac{d s}{\pi \sqrt{1-s^{2}}} \frac{(1+a t) d t}{\pi \sqrt{1-t^{2}}} .
$$

According to Corollary 7 there exist a constant $C>0$, not depending on $n$, and $N_{1} \leq 2 C n$ points $t_{1}, \ldots, t_{N_{1}}$ which are the nodes of a Chebyshev-type quadrature formula for $w_{-a}(t)$ of degree $2 n$. There also exist $n+1$ points $s_{1}, \ldots, s_{n+1}$ which are the nodes of a Chebyshev-type quadrature for $w_{0}(t)$ of degree $2 n$. (Simply take the nodes of the $(n+1)$-point Gauss-Chebyshev quadrature formula.) Then it is easy to see that

$$
\int_{-1}^{1} \int_{-1}^{1} p(s, t) \frac{d s}{\pi \sqrt{1-s^{2}}} \frac{(1+a t) d t}{\pi \sqrt{1-t^{2}}}=\frac{1}{N_{1}(n+1)} \sum_{i=1}^{n+1} \sum_{j=1}^{N_{1}} p\left(s_{i}, t_{j}\right)
$$

holds for every polynomial $p(s, t)$ of degree $\leq 2 n$. Take $\phi_{i}=\arccos s_{i}$, $\psi_{j}=\arccos t_{j}$, and

$$
x_{i j}=\cos \phi_{i}\left(1+a \cos \psi_{j}\right), \quad y_{i j}=\sin \phi_{i}\left(1+a \cos \psi_{j}\right), \quad z_{i j}=a \sin \psi_{j} .
$$


Then

$$
\frac{1}{4 \pi^{2} a} \iint_{T_{a}} f(x, y, z) d \sigma=\frac{1}{N_{1}(n+1)} \sum_{i=1}^{n+1} \sum_{j=1}^{N_{1}} f\left(x_{i j}, y_{i j}, z_{i j}\right)
$$

holds for all polynomials $f(x, y, z)$ of degree $\leq n$ which are even in $y$ and $z$. By the symmetry in $y$ and $z$, it then follows that the $4 N_{1}(n+1)$ points

$$
\left(x_{i j}, \pm y_{i j}, \pm z_{i j}\right), \quad i=1, \ldots, n+1, \quad j=1, \ldots, N_{1} \text {, }
$$

are the nodes of a Chebyshev-type quadrature formula of size $\leq 8 C n(n+1)$ and degree $n$.

\section{BIBLIOGRAPHY}

1. J.D. Buckholtz, A characterization of the exponential series, Amer. Math. Monthly 73 Part II, (1966), 121-123.

2. K.-J. Förster, Variance in quadrature-a survey, Numerical Integration IV (H. Brass and G. Hämmerlin, eds.), ISNM vol. 112, Birkhäuser, Basel, 1993, pp. 91-110.

3. W. Gautschi, Advances in Chebyshev quadrature, Numerical Analysis (G.A. Watson, ed.), Lecture Notes in Math., vol. 506, Springer, Berlin, 1976, pp. 100-121.

4. Ja.L. Geronimus, On the Čebyšev quadrature formula, Izv. Akad. Nauk SSSR Ser. Mat. 33 (1969), 1182-1207; English transl. in Math. USSR-Izv. 3 (1969), 1115-1138.

5. L.L. Helms, Introduction to potential theory, Wiley-Interscience, New York, 1969.

6. J. Korevaar and J.L.H. Meyers, Chebyshev-type quadrature on multidimensional domains, Univ. of Amsterdam, Math. preprint series, Report 93-01, 1993; J. Approx. Theory 79 (1994), 144-164.

7. A.B.J. Kuijlaars, The minimal number of nodes in Chebyshev type quadrature formulas, Indag. Math. (N.S.) 4 (1993), 339-362.

8. F. Peherstorfer, Weight functions which admit Tchebycheff quadrature, Bull. Austral. Math. Soc. 26 (1982), 29-37.

9. __ On Tchebycheff quadrature formulas, Numerical Integration III $(\mathbf{H}$. Brass and G. Hämmerlin, eds.), ISNM vol. 85, Birkhäuser, Basel, 1988, pp. 172-185.

10. G. Pólya and G. Szegö, Aufgaben und Lehrsätze aus der Analysis I, Springer, Berlin, 1925.

11. P.D. Seymour and T. Zaslavsky, Averaging sets: a generalization of mean values and spherical designs, Adv. in Math. 52 (1984), 213-240.

12. A.H. Stroud, Approximate calculation of multiple integrals, Prentice-Hall, Englewood Cliffs, NJ, 1971.

13. G. Szegö, Über eine Eigenschaft der Exponentialreihe, Sitzungsber. Berl. Math. Ges. 23 (1924), 50-64; reprinted in Collected Papers - Volume I, Birkhäuser, Boston, 1982, pp. 645660.

14. Math. Soc., Providence, RI, 1975.

15. R.S. Varga, Scientific computation on mathematical problems and conjectures, CBMS vol. 60 , SIAM, Philadelphia, PA, 1990.

16. Y. Xu, Weight functions for Chebyshev quadrature, Math. Comp. 53 (1989), 297-302.

Faculteit Wiskunde en Informatica, Universiteit van Amsterdam, Plantage MuiderGRACHT 24, 1018 TV AMSTERDAM, THE NETHERLANDS

E-mail address: arno@fwi.uva.nl 